\title{
LA FORMACIÓN DE LOS HIPOCORÍSTICOS EN EL ESPAÑOL DE MÉXICO
}


Los hipocorísticos son un tipo de fenómeno perteneciente a los truncamientos que se puede encontrar en casi todas las lenguas. En el español, dicho fenómeno da pie a que un nombre propio derive en uno o más hipocorísticos que, pese a lo que se había manejado tradicionalmente, son originados por mecanismos bien específicos y no son meras deformaciones arbitrarias. Pueden ser éstos de tipo prosódico, silábico, segmental o morfológico. En el presente trabajo se hizo una recopilación de algunos de los hipocorísticos más comunes en México y se analizaron los mecanismos presentes en ellos. Posteriormente se ordenaron estadísticamente para poder encontrar patrones en los mismos y observar si variables como la edad o el sexo pueden establecer diferencias en la selección de un determinado mecanismo.

Palabras clave: hipocorístico, truncamiento, mecanismos prosódicos, mecanismos segmentales, palatalización, oclusivización

Hypocoristics are a type of phenomenon belonging to truncations that can be found in almost all languages. In Spanish, this phenomenon gives rise to a proper name to derive in one or more hypocoristics that despite what had traditionally been handled, are caused by very specific mechanisms and are not mere arbitrary deformations. Such mechanisms are prosodic type, syllabic, segmental or morphological. In this paper we made a compilation of some of the most common hypocoristics in Mexico and mechanisms present in them were analyzed. They were then statistically ordered to find patterns in the mechanisms and see if variables such as age or sex may differentiate in the selection of a particular one.

KeY wORDS: hypocoristic, truncation, prosodic mechanisms, segmental mechanisms, palatalization, oclusivization 


\section{LA FORMACIÓN \\ DE LOS HIPOCORÍSTICOS \\ EN EL ESPAÑOL \\ DE MÉXICO}

Juan Bernardo Estrada

\section{Introducción}

Los hipocorísticos son un tipo de acortamiento muy usado en nuestro país. Colina (1996) señala que: "Spanish hypocoristic formation and noun truncation consist in copying the first two syllabes of the base and moving the stress to the first syllabe of the truncated form". Aunque esta definición es cuestionable, vemos que nombres como Federico, Alejandra, Candelario, Catalina y Lucila pueden originar los respectivos hipocorísticos Fede, Ale, Cande, Cata y Luci en nuestra lengua. Son tan comunes, que en ocasiones los hablantes ya no recuerdan el nombre propio del cual se han formado. El tema de los hipocorísticos ya ha sido tratado por diversos autores como Boyd-Bowman (1955), Wijk (1964), Urawa 
(1985), Prieto (1992), Báez (2002) y Gutiérrez (2009). Salvo el trabajo de Espinosa (2001), en el que se hace una recopilación de procesos fonéticos, y el de Gutiérrez (2009), en el que se analizan los procesos fonológicos, los estudios no se han centrado en los mecanismos de formación de hipocorísticos.

Dentro de los distintos mecanismos de formación de hipocorísticos en español, existen cuatro grandes bloques: prosódico, segmental, silábico y morfológico. Cada uno de ellos, a su vez, comprende varios mecanismos: el truncamiento, el reacomodo acentual, la adición de género, la palatalización, la adición de -i, la simplificación de inicio complejo, etc. Tomando un nombre como Salvador y su hipocorístico Chava pueden desglosarse los mecanismos presentes en tal fenómeno.

\begin{tabular}{|l|c|c|c|c|}
\hline \multicolumn{5}{|c|}{ TIPOS DE MECANISMOS } \\
\hline & $\begin{array}{c}\text { Pruncamiento } \\
\text { silábico }\end{array}$ & $\begin{array}{c}\text { Reasignación } \\
\text { acentual }\end{array}$ & $\begin{array}{c}\text { Elisión } \\
\text { de coda }\end{array}$ & Palatalización \\
\hline Salvador $>$ & {$[$ sal. $\beta \mathrm{a}]>$} & {$[$ 'sal. $\beta \mathrm{a}]>$} & {$[$ 'sa. $\beta \mathrm{a}]>$} & $[\mathrm{t}] \mathrm{a} . \beta \mathrm{a}]$ \\
\hline
\end{tabular}

Este trabajo está enfocado no tanto en los hipocorísticos, sino en los mecanismos como los que acabo de mostrar. ${ }^{1} \mathrm{Se}$ hizo una recopilación de hipocorísticos en varias ciudades del país y, a partir de ello, se clasificaron dentro de los cua-

\footnotetext{
${ }^{1}$ Si me decidí únicamente por los procesos de los hipocorísticos fue porque aquellos que se presentan en los nombres comunes no son tan variados. Es casi una regla que sólo se acorta el nombre común mediante apócope: refrigerador $\rightarrow$ refri, película $\rightarrow$ peli, suburbano $\rightarrow$ subur, tranquilo $\rightarrow$ tranqui, etc.
} 
tro bloques ya mencionados. Por lo tanto, la importancia de este trabajo radica en la exposición detallada y clasificación de los mecanismos formadores de hipocorísticos.

Este artículo se divide en tres apartados. En el primero se dará un panorama teórico sobre el truncamiento y los mecanismos creadores de hipocorísticos, ya que éste es considerado, en algunos casos, como una variante del primero. En el segundo se explicará la metodología del estudio llevado a cabo: la recopilación del corpus, la selección de las ciudades objeto de estudio y la aplicación del ejercicio para obtener los datos. Finalmente, en un tercer apartado se describirán todos los mecanismos encontrados en México y cómo fueron clasificados.

\section{Truncamiento e hipocorísticos}

Casado Velarde define el acortamiento (lo que yo denominaré, a partir de este punto, nombre común trunco, ya que sólo a este tipo de sustantivos se refiere su definición) como "el resultado de un proceso mediante el cual una unidad léxica, simple o compleja, ve reducido su significante reteniendo el mismo significado y categoría gramatical (clase de palabra)" (1999: 5077). Para este autor el truncamiento es un fenómeno que no afecta más que la prosodia de la palabra.

Por su parte, Prieto (1992) asegura: "Basically, Hypocoristic Formation and Noun Truncation consist in copying the first two syllabes of the base name - with some interesting restrictions $[\ldots]$ and movement of the stress to the first syllabe, forming what we might call a trochaic pattern" (las cursivas son mías). 
Algo que llama la atención en ambas definiciones es la sencillez con la que parece darse el fenómeno del acortamiento. Para ambos autores dicho fenómeno únicamente consiste en acortar palabras; en el caso de Prieto, tal sencillez parece alcanzar los hipocorísticos. Esto resulta muy significativo, porque a lo largo de esta investigación recopilé una gran cantidad de mecanismos presentes en la formación de hipocorísticos y que al parecer ningún autor menciona en sus definiciones. Así pues, al menos en el caso de los hipocorísticos, mostraré que no es un proceso tan sencillo.

También se ha afirmado que un hipocorístico "es un vocablo usado con intención afectuosa, que a veces ha sido sometido a cierta deformación [y que sirve para aludir] especialmente a las abreviaturas y modificaciones que sufren los nombres propios" (Carreter apud Báez, 2002: 242). El uso del término deformaciones puede ser cuestionable, ya que son más bien modificaciones sistemáticas (tal vez equiparables con los mecanismos que describiré más adelante).

Boyd-Bowman opina que "los hipocorísticos, lejos de ser, como parecería a primera vista, meras sustituciones arbitrarias, responden a principios fonéticos no menos regulares que muchos de los que conoce la lengua general", y continúa, "estas deformaciones se deben en gran parte al rudimentario sistema fonético de los niños que aprenden a hablar, y a los esfuerzos conscientes que hacen los adultos con intención cariñosa para imitar ese sistema" (1955: 337-338). Por tanto, los hipocorísticos no son propiamente deformaciones, pero sí atienden a tales procesos lingüísticos (principios fonéticos) y que además pueden ser un acto originado por un adulto o por un niño. 
Una última definición de los hipocorísticos dice que "en la mayoría de estas reducciones se observa que el apodo resultante es una palabra de dos sílabas, y éstas son siempre el prototipo silábico universal consonante+vocal, salvo cuando el nombre propio tiene una sílaba final trabada por consonante nasal [...]. Además, los apodos surgen con acentuación llana, aún si el nombre original contiene acentuación grave o esdrújula" (Núñez y Morales, 1999: 63).

Algunas de las definiciones dadas coinciden en el hecho de que los hipocorísticos son palabras bisílabas. Otras coinciden en utilizar el término deformaciones (como Carreter y Boyd-Bowman en las definiciones ya dadas). Sin embargo, en ninguna de ellas se menciona de manera explícita los tipos de mecanismos a los que aludí en la introducción. Considero que una definición más completa de hipocorístico debería incluir tales mecanismos. Por lo tanto, sostengo que un hipocorístico es una palabra, por lo general bisílaba y llana, con una fuerte carga afectiva y que ha sido modificada en cuanto a su cantidad silábica, su acentuación, sus límites silábicos o su pronunciación, entre otras características, por mecanismos de tipo prosódico, segmental, silábico o morfológico, con algunas particularidades.

Son varios los mecanismos que, a partir de un nombre propio, originan los hipocorísticos. Entre los principales -y que además han sido mencionados pero no explicados sistemáticamente por los autores citados anteriormente- pueden encontrarse los siguientes:

1. Truncamiento silábico. Consiste en eliminar una o varias sílabas, ya sea en el inicio, al interior o al final de una 
palabra. En el truncamiento el interés no está tanto en aquello que se elimina sino en lo que se conserva. La mayoría de los hipocorísticos presentan truncamiento silábico, salvo aquellos casos en que el nombre ya es bisílabo o monosílabo. En español pueden truncarse los nombres propios en casi cualquier sílaba. Según Prieto (1992) y Colina (1996) es más frecuente el truncamiento a partir de la sílaba inicial: es decir, que permanecen las dos primeras sílabas. De acuerdo con Sempere (2006) hay tres tipos de truncamiento: en el que se elimina material fónico del lado izquierdo (Tipo I); en el que se elimina el del lado derecho (Tipo II) y aquel caso en que se elimina material de ambos lados de la palabra (Tipo III).

2. Reasignación de acento. Con excepción de los truncamientos a partir de sílaba inicial y acentuada, la mayoría de los hipocorísticos han pasado por este mecanismo para alcanzar el esquema de acentuación llano. Ohannesian ya había mencionado que algunos hipocorísticos se forman " $[\ldots]$ independientemente de la posición de la sílaba tónica, sea la base paroxítona [...], oxítona [...] o proparoxítona [...]" (2004: 57).

3. Simplificación de inicio complejo y coda silábica. Cuando las sílabas de los nombres propios poseen un esquema silábico $\mathrm{CCV}(\mathrm{C})$ o $(\mathrm{C}) \mathrm{VC}$ suele recurrirse a "[la] simplificación de grupos consonánticos y la conversión de sílabas trabadas en libres" (Báez, 2002: 258). Suele suceder algo similar en el núcleo silábico en el que existe un diptongo: se simplifica quedando sólo una vocal.

4. Reduplicación silábica. Este mecanismo se considera derivado del proceso de aprendizaje del habla infantil. 
Es común que "cuando el niño trata de dar cuerpo a la palabra o de reforzar el sonido suele repetir la misma sílaba" (Jakobsen apud Boyd-Bowman, 1955: 347).

5. Adición de -i. Aunque Urawa (1985) y algunos otros autores consideran que es una terminación cariñosa que se da en los préstamos del inglés, es un mecanismo muy recurrente en español.

6. Palatalización. Al formar los hipocorísticos en español, "se convierte en $c h$ toda $s$ que no llegue a perderse en final de sílaba" (Boyd-Bowman, 1955: 346). Por lo tanto, es un mecanismo que se considera común.

7. Oclusivización. También se considera un mecanismo derivado del habla infantil, debido a que los niños aprenden primero los fonemas oclusivos y luego los fricativos; por tanto, hay una tendencia de los infantes a oclusivizar todo fonema que no lo sea. Este mecanismo se menciona en los trabajos de Boyd-Bowman (1955) y Wijk (1964).

Es preciso mencionar que a lo largo de mi trabajo encontré estos mecanismos y algunos otros que serán explicados detalladamente en el tercer apartado. En una primera clasificación, pueden agruparse de la siguiente manera (cuadro 1):

Cuadro 1. Clasificación de mecanismos mencionados en diversos autores

\begin{tabular}{|l|l|l|l|}
\hline \multicolumn{1}{|c|}{ Prosódicos } & \multicolumn{1}{|c|}{ Silábicos } & \multicolumn{1}{c|}{ Segmentales } & Morfológicos \\
\hline $\begin{array}{l}\text { Truncamiento } \\
\text { Reasignación } \\
\text { acentual }\end{array}$ & $\begin{array}{l}\text { Eliminación de coda } \\
\text { compleja } \\
\text { Simplificación de inicio } \\
\text { complejo }\end{array}$ & $\begin{array}{l}\text { Palatalización } \\
\text { Oclusivización } \\
\text { Reduplicación }\end{array}$ & Adición de -i \\
\hline
\end{tabular}




\section{Metodología}

Para poder recopilar y clasificar los mecanismos de los que hablaré en el tercer apartado fue necesario realizar una investigación de campo. Con ésta se extrajo una cantidad considerable de datos que se analizaron y que me ayudaron a reconocer dichos mecanismos.

Se preparó un corpus de 204 nombres propios ${ }^{2}$ extraídos principalmente de tres fuentes: del calendario Galván en su edición de 2010, del artículo de Báez, Herrera y Mendoza (1993) titulado "Antropónimos en el español de la ciudad de

\footnotetext{
${ }^{2}$ Los nombres a estudiar fueron los siguientes: Adelaida, Adriana, Agustín, Alberto, Alejandra, Alejandro, Alfonso, Alfredo, Alicia, Anabel, Anastasio, Andrea, Andrés, Ángeles, Angélica, Anselmo, Antonieta, Antonio, Araceli, Armando, Arturo, Ascensión, Asunción, Azucena, Bárbara, Beatriz, Benito, Benjamín, Berenice, Bernardo, Bonifacio, Camilo, Candelario, Carlos, Carmen, Carolina, Catalina, Cayetano, Cecilia, Christian, Claudia, Concepción, Consuelo, Cresencio, Cristina, Cristóbal, Cuauhtémoc, Delfina, Dolores, Domingo, Donaciano, Edmundo, Eduardo, Efraín, Eleazar, Elisa, Eliseo, Elizabeth, Emeterio, Emilio, Emmanuel, Enrique, Epifania, Ernestina, Ernesto, Esperanza, Estanislao, Esteban, Eufrasia, Eustaquio, Evaristo, Evelia, Ezequiel, Fabián, Fabricio, Fausto, Federico, Feliciano, Felipe, Fermín, Fernanda, Fernando, Florentina, Francisca, Francisco, Fulgencio, Gabriel, Gabriela, Genoveva, Georgina, Gerardo, Gilberto, Gisela, Graciela, Gregorio, Guadalupe, Guillermo, Gustavo, Heraclio, Heriberto, Hilario, Humberto, Ignacio, Inocencio, Isabel, Isidro, Israel, Jacqueline, Jazmín, Jennifer, Jessica, Jesús, Joaquín, Jonathan, Jorge, José, Josefina, Juan, Julieta, Juventino, Karina, Katia, Ladislao, Leonardo, Leonor, Leopoldo, Leticia, Liliana, Lissete, Lizbeth, Lorena, Lorenzo, Lourdes, Lucila, Luis, Magdalena, Manuel, Marcela, Margarita, María, Maribel, Marisela, Marisol, Matilde, Maximiliano, Maximino, Miriam, Moisés, Mónica, Montserrat, Natalia, Nicolás, Oliver, Paola, Patricia, Paula, Paulina, Paulo, Pedro, Rafael, Ramón, Ramona, Raquel, Raúl, Raymundo, Refugio, Regina, Reynaldo, Ricardo, Roberto, Rocío, Rogelio, Rosa, Rosalío, Rosario, Rosendo, Roxana, Salvador, Samanta, Samuel, Sandra, Santiago, Sebastián, Sergio, Socorro, Sofía, Soledad, Susana, Teresa, Timoteo, Tomás, Ulises, Valentín, Valeria, Vanessa, Verónica, Vicente, Víctor, Virginia, Viridiana, Viviana, Yadira, Yolanda, Yuridia.
} 
México. Tradición y novedad", y la tesis doctoral de Gutiérrez Santana (2009) titulada "Procesos fonológicos utilizados en la formación de hipocorísticos." Se eligieron los nombres con base en las coincidencias entre el artículo de Báez, Herrera y Mendoza y la tesis de Gutiérrez. Ambos textos trabajan con nombres de uso corriente en México, lo que me permitió tomarlos como "usuales" o de mayor distribución. A lo anterior habría que sumar el calendario del que se obtuvieron, el Galván, del que se tomaron algunos nombres ya casi en desuso, pero que han generado hipocorísticos muy comunes en nuestra lengua y que, en mi opinión, deben ser incluidos para poder recopilar los mecanismos.

Una vez delimitado el corpus de trabajo, se seleccionaron tanto los informantes como las ciudades donde se haría la investigación de campo. Se eligieron cuatro informantes por localidad, que hubieran nacido en la ciudad objeto de estudio y que hubieran radicado la mayor parte de su vida ahí mismo. Esto con la finalidad de que los datos obtenidos no se vieran contaminados por un habla distinta a la de su ciudad.

En cuanto a las ciudades en las que se realizó la investigación de campo, se buscó que cubrieran las zonas dialectales de nuestro país. Si bien es cierto que hay discrepancias sobre dichas zonas, sí hay coincidencias en cuanto a algunas regiones, como Chiapas, Yucatán, el norte y el centro del país. Por tanto, tomando en cuenta las diversas zonas dialectales que han propuesto autores como Henríquez Ureña (1921), Moreno de Alba (1994) y Serrano (2002), elegí las que se muestran en el mapa 1 como representantes de dichas zonas de México. En algunos casos, debido a la amplitud de 
la zona, se seleccionó más de una ciudad que cubriera esa región. ${ }^{3}$

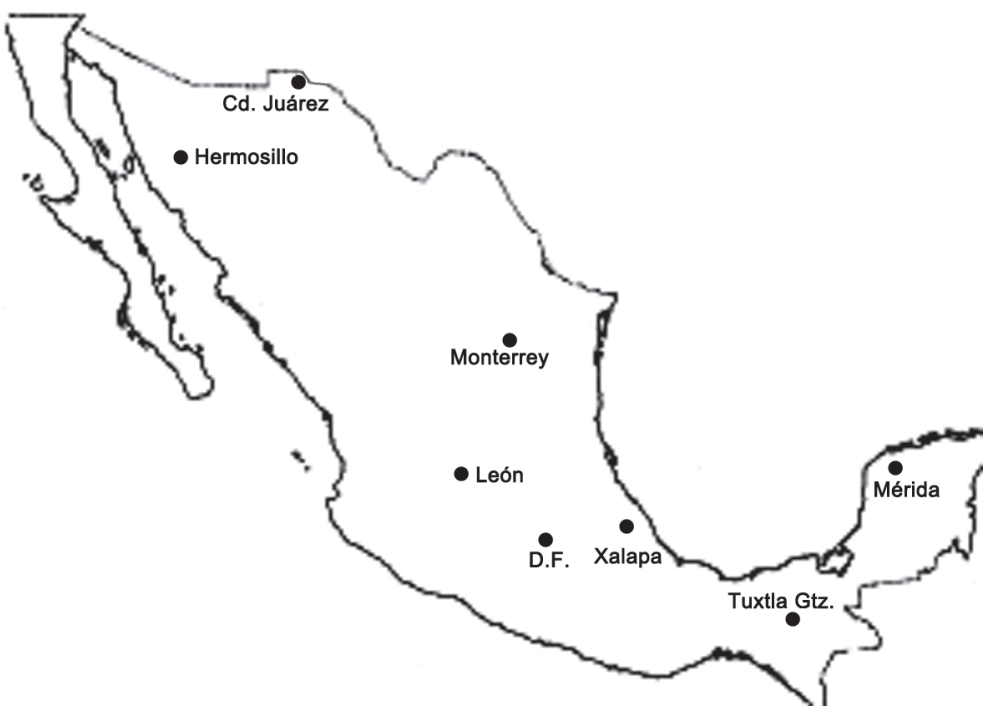

Mapa 1. Ciudades en las que se llevó a cabo la investigación

${ }^{3}$ a) Monterrey, Ciudad Juárez y Hermosillo. La parte norte de nuestro país es una zona muy amplia. Por esa razón decidí hacer una subdivisión y elegir una ciudad del noreste (Monterrey), y otra del noroeste (Hermosillo). Además, por su cercanía con Estados Unidos, elegí Ciudad Juárez.

b) Distrito Federal. Las razones son obvias, todo estudio que abarque una gran parte del territorio nacional tiene que considerar la capital, como muestra del dialecto central.

c) Mérida. Elegí esta ciudad por ser la capital del estado y la mayor de la región peninsular. Tres de las propuestas que he mostrado en este apartado consideran a Yucatán como una región dialectal.

d) León. En el estudio de Serrano (2002), se considera que los estados de Guanajuato, Jalisco, Michoacán, entre otros, pertenecen a una variante occidental (criterio perceptual). Moreno de Alba (1994) los considera parte del dialecto central (criterio fónico). Si me decidí por la ciudad de León, en lugar de la capital del estado, fue por su ubicación como principal centro urbano y económico de la región.

e) Xalapa. El estado de Veracruz abarca, según Henríquez Ureña (1921), dos zonas dialectales, considerando una división entre sus tierras bajas y altas; según 
Los 204 nombres fueron agrupados en una presentación. A cada informante se le mostró en una pantalla de computadora dicha presentación y se le pidió que dijera el hipocorístico (previa explicación del término) que conociera para cada nombre. Dichas entrevistas fueron grabadas en la computadora mediante el programa Praat (versión 5.2.13) con un micrófono USB Steren (modelo COM-080) especial para registro de voz. En la medida de lo posible, se buscó que las entrevistas fueran realizadas en el domicilio del informante, primero, para que tuviera mayor confianza en su propio entorno; y segundo, para tener un lugar apropiado, libre de ruidos que pudieran interferir en las grabaciones. Una vez obtenidos los datos, se acomodaron de manera individual para determinar qué mecanismos eran los más utilizados por los hablantes. En el siguiente apartado se muestran los resultados.

\section{Mecanismos de formación de hipocorísticos usados en México}

En el primer apartado se describieron los tipos de mecanismos agrupados en cuatro grandes bloques: prosódico (es decir, los que afectan a la palabra, ya sea en su extensión

Serrano (2002) sólo abarca una: la costeña; Lope Blanch (1990) reconoce un dialecto veracruzano meridional y uno norteño. Nuevamente encontré dificultad al delimitar esta región. Por esto, me incliné por la capital del estado.

f) Tuxtla Gutiérrez. Dos de los autores que revisé toman a Chiapas como una zona dialectal aparte. Para González Moreno (1935) está en una región genérica que él denomina suroccidental. Como sea, este estado posee una gran cantidad de lenguas indígenas, lo que lo hace aún más peculiar, y debe ser estudiado como una entidad separada. 
o en su acentuación); silábico (aquellos que modifican las estructuras silábicas: el ataque, el núcleo o la coda); segmental (los que afectan cada uno de los fonemas segmentales) $\mathrm{y}$, finalmente, morfológico (los que provocan cambios en la morfología y añaden o modifican sufijos). Aquí se describirán todos los mecanismos encontrados a lo largo de la investigación de campo y la recopilación de datos.

\section{Mecanismos prosódicos}

En español, los principales mecanismos de tipo prosódico que pude localizar como creadores de hipocorísticos, fueron los siguientes.

\section{Truncamiento}

Encontré que el truncamiento puede darse casi en cualquier sílaba de la palabra, o ser de tres tipos distintos, de acuerdo con Sempere (2006). Parece que uno de los más frecuentes es el que se da a partir de la sílaba inicial que, además, puede estar acentuada o no. En el cuadro 2 reúno algunos ejemplos.

Cuadro 2. Truncamiento en sílaba inicial

\begin{tabular}{|c|c|}
\hline $\begin{array}{l}\text { Truncamiento en sílaba inicial átona } \\
\text { (requiere de reasignación de } \\
\text { acento) }\end{array}$ & $\begin{array}{l}\text { Truncamiento en sílaba inicial tónica } \\
\text { (no requiere de reasignación de } \\
\text { acento) }\end{array}$ \\
\hline $\begin{array}{l}\text { [a.le.'xan.dra] }>\text { ['a.le] Alejandra } \\
\text { [xe.no.'ße. } \beta a />[\text { 'xe.no] Genoveva } \\
\text { [be.'ni.to] }>[\text { 'be.ni] Benito } \\
\text { [kan.de.'la.rjo] }>\text { ['kan.de] Candelario }\end{array}$ & $\begin{array}{l}\text { ['mo.ni.ka] }>\text { ['mo.ni] Mónica } \\
\text { ['je.si.ka] }>\text { ['je.si] Jessica } \\
\text { ['o.li.ßer] }>\text { ['o.li] Oliver } \\
\text { ['jo.na.tan] }>\text { ['jo.na] } ~[\text { 'jo.ni] Jonathan }\end{array}$ \\
\hline
\end{tabular}


También se puede presentar el truncamiento a partir de la sílaba tónica (descartando la inicial tónica que acabo de mostrar en el cuadro 2), lo que además puede conllevar una serie de mecanismos, no así la reasignación acentual. A continuación (cuadro 3) se muestra este tipo de truncamiento. ${ }^{4}$

Cuadro 3. Truncamiento en sílaba tónica

\begin{tabular}{|l|l|}
\hline $\begin{array}{l}\text { Truncamiento en sílaba tónica } \\
\text { (antepenúltima sílaba) }\end{array}$ & $\begin{array}{l}\text { Truncamiento en sílaba tónica final } \\
\text { (última sílaba) }\end{array}$ \\
\hline $\begin{array}{l}\text { [ra.'mo.na] }>\text { ['mo.na] Ramona } \\
\text { [xu.ßen.ti.no] }>\text { ['ti.no] Juventino } \\
\text { [al.'ßer.to] }>\text { ['be.to] Alberto }\end{array}$ & $\begin{array}{l}\text { [ra.mon] }>[\text { mon.'tfo] Ramón } \\
\text { [a.sun.sion] }>\text { ['tfo.na] Asunción } \\
\text { [be.a.tris] }>\text { ['ti.tfi] Beatriz }\end{array}$ \\
\hline
\end{tabular}

Aunque es menos frecuente, también se presenta en español el truncamiento en una sílaba diferente a la inicial o a la acentuada (véase cuadro 4 en la página siguiente).

\section{Reasignación de acento}

Este mecanismo está muy ligado al anterior, ya que, como se observa, en muchas ocasiones, los truncamientos requieren

\section{${ }^{4}$ Derivación del diminutivo}

Como parte del mecanismo de truncamiento se dio este caso particular. En mi investigación sólo lo encontré en nombres masculinos, sin embargo, ello no indica que sea restrictivo en cuanto al género. Se dieron muy pocos ejemplos, pero de cualquier manera los muestro.

Formación de hipocorísticos a partir del diminutivo

Nombre

Gilberto

Ernesto

Alberto

Luis
Diminutivo

Gilbertito

Ernestito

Albertito

Luisito
Hipocorístico

['ti.to] Tito

['ti.to] Tito

['ti.to] Tito

['tfi.to] Chito 
Cuadro 4. Truncamiento en otra sílaba

\begin{tabular}{|c|c|}
\hline Se acortan sin tomar la sílaba tónica & $\begin{array}{c}\text { Se acortan en la sílaba pretónica } \\
\text { y hacen cambio acentual }\end{array}$ \\
\hline [e.li.sa.'ßet] $>$ ['li.sa] Elizabeth ${ }^{*}$ & [i.sa:'ßel] $>\left[{ }^{‘} \mathfrak{t}\right.$ a. $\left.\beta \mathrm{e}\right]$ Isabel ${ }^{* *}$ \\
\hline [an.xe.li.ka]>['ki.ka] Angélica & [a. $\gamma$ us.tin] $]>\left[{ }^{\prime} \gamma\right.$ u.ti] Agustín \\
\hline \multirow[t]{2}{*}{ [es.ta.nis.'la.o]>['ta.nis] Estanislao } & [e.se.'kjel] $>$ ['tfe.ke] Ezequiel \\
\hline & [es.pe.'ran.sa]>['pe.ra] Esperanza \\
\hline
\end{tabular}

de una reasignación acentual (excepto cuando el hipocorístico se forma a partir de la sílaba tónica). Ambos menismos van de la mano, puesto que el objetivo de esto es que la forma resultante sea un pie trocaico. Es común que los hipocorísticos que surgen de un truncamiento a partir de la sílaba inicial no acentuada deban reasignar el acento: [ba.'le.rja] $>$ ['ba.le] Valeria, [ba.'ne.sa] $>$ ['ba.ne] Vanessa, [e.le.a.sar] $>$ ['e.le.a] ['e.lea] Eleazar. Lo mismo sucede cuando el hipocorístico surge a partir del truncamiento de una sílaba que no sea ni la inicial ni la acentuada: [an.to.nje. ta] $>$ ['to.ni] Antonieta, [ro.sa.'li.o] $>$ ['t fa.lo] Rosalío. Esto da como resultado una palabra bisílaba y llana sin importar dónde se haya encontrado el acento en el antropónimo.

\footnotetext{
* En este caso, quizá se forme de esta manera por su similitud con el nombre de Elisa. Lo mismo podría decirse del caso de Antonieta, cuyo hipocorístico ['to.ni] tal vez surja por el nombre de Antonia.

** En los casos de Isabel, Ezequiel y Agustín es posible que se prefiera tomar las dos últimas sílabas, en lugar de hacer el truncamiento a partir de la sílaba acentuada (que también es la final) porque tendría que añadirse una vocal protética. Desde el punto de vista de la economía del lenguaje es mucho más factible eliminar que añadir.
} 


\section{Elisión de vocal final}

Este fenómeno había sido ya descrito por Boyd-Bowman (1955) como perteneciente a los hipocorísticos yucatecos, influenciados por la lengua maya en la región. Otros investigadores señalan la presencia de consonantes finales no típicas en el castellano. Dice Lope Blanch: "La aparición de varios sonidos consonánticos en posición final de palabra (-p, $-t,-k,-c ̌,-s ̌ s,-\hat{s})$ también en lexemas de procedencia maya [...]. Y la propagación de esos finales consonánticos, anómalos en castellano, a voces de origen hispánico (cambalach, coch: Suárez pág. 51) sí representaría un caso de penetración fonética maya" (1981: 417). De esta manera, resultan hipocorísticos como los del siguiente cuadro:

Cuadro 5. Hipocorísticos con elisión vocálica producidos en Mérida

\begin{tabular}{|l|c|c|}
\hline Nombre & Hipocorístico bisílabo & Hipocorístico con elisión \\
\hline Fabián & ['fa.ßi] & ['faß] \\
\hline Hilario & ['i.la] & ['il] \\
\hline Isidro & ['i.si] & ['is] \\
\hline Katia & ['ka.ti] & ['kat] \\
\hline Matilde & ['ma.ti] & ['mat] \\
\hline Dolores & ['lo.lo] ['lo.la] & ['lol] \\
\hline
\end{tabular}

También en Mérida ${ }^{5}$ se registraron algunos hipocorísticos muy peculiares, que presentan características como las que mencionaba Lope Blanch (1981): ['grek] Gregorio, ['ritf]

\footnotetext{
${ }^{5}$ En algunas otras ciudades también se reportó este fenómeno que, no obstante, quizá se debió a otras causas. Por ejemplo, los siguientes hipocorísticos ['maks] Maximiliano, ['roks] Roxana, ['jes] Jessica parecen préstamos del inglés. No podría yo asegurar que a ello se deba su elisión vocálica, sin embargo sí llama la atención el fenómeno.
} 
Ricardo, ['rotf] Rogelio, [tu.'rif] Arturo. Ni /k/, /tg/ o / $/$ se encuentran en posición final de palabra en castellano. Además, el último fonema ni siquiera se encuentra dentro del inventario de los fonemas de nuestra lengua. Por lo tanto, debe ser la influencia de la lengua maya la causa de estos fenómenos.

\section{Adición de sílaba -ch(o)}

En dos casos se añadió una partícula -ch-, además de un morfema de género $-o$, lo que dio como resultado la sílaba -cho: ['xuan. t5o] Juan y ['mon.t5o] Ramón. Al parecer su función está encaminada a aumentar la cantidad silábica para completar el esquema bisílabo de la palabra mínima en español. Desafortunadamente no fue un proceso tan frecuente para poder ofrecer una explicación más adecuada.

\section{Mecanismos silábicos}

En mi investigación encontré alrededor de cinco mecanismos silábicos, sin embargo sólo mostraré los tres que se presentaron con mayor frecuencia.

Elisión de coda compleja

Con excepción del fonema nasal alveolar /n/ en posición de coda silábica, que por lo general permanece al formar el hipocorístico, las consonantes tienden a desaparecer debido a la condición de coda (cuadro 6).

\footnotetext{
${ }^{6}$ Cumplen la coda condition las consonantes sin punto de articulación (como el saltillo / $/$ / y la aspiración /h/) y aquellas consonantes cuyo punto de articulación les es heredado por asimilación de la consonante siguiente. Tal es el caso, por ejemplo, de la /n/ de Poncho, cuya articulación se asimila a la posalveolar que le
} 
Cuadro 6. Hipocorísticos con elisión de coda

\begin{tabular}{|l|c|}
\hline \multicolumn{1}{|c|}{ Nombre } & Hipocorístico con elisión de coda \\
\hline Magdalena & [ma.'le.na] \\
\hline Virginia & ['bi.ki] \\
\hline Esperanza & ['pe.ra] \\
\hline Lorenzo & ['lo.re] \\
\hline Anselmo & ['tfe.mo] \\
\hline Valentín & ['ba.le] \\
\hline
\end{tabular}

Salvo el caso de ['pe.ra] "Esperanza”, en el que se elide la coda de una sílaba átona, en los demás ejemplos del cuadro 6 la elisión se da en la sílaba tónica. También hay hipocorísticos de estos mismos nombres en que se conservan las consonantes en coda: ['maү.ða] Magdalena, ['bir.xi] Virginia, [pe.'lan.tfa] Esperanza, ['lo.ren] Lorenzo, ['sel.mo] Anselmo, ['ba.len] Valentín, etc.

\section{Simplificación de inicio complejo}

En el ataque silábico en español suelen aparecer grupos bifonemáticos consonánticos, conformados por oclusivos y fricativo labiodental + líquida $($ salvo $/ d /+/ l /)$. Debido al Principio de dispersión sonora de Clements (1990) (una consonante en inicio es mejor en tanto tenga menor sonoridad), al formarse un hipocorístico a partir de un nombre que contenga un grupo bifonemático, permanece siempre la consonante no líquida. Por lo tanto, resulta común escuchar hipocorísticos como ['pa.ti] Patricia, ['ga.ßi] Gabriela, ['be.ti] ['ti.tfi]

sigue. Las consonantes que no tienen a qué consonante asimilarse, o las que no son susceptibles de hacerlo, no cumplen con la coda condition, y por ello es más probable que se pierdan. 
Beatriz, ['pan.t5o] Francisco, ['fe.jo] Alfredo, ['kaja] Claudia, ['fa.ßi] Fabricio, etc.

\section{Monoptongación}

De acuerdo con el patrón silábico universal, CV, es mejor evitar la complejidad en cualquier parte de la sílaba, incluyendo el núcleo. Es por ello que, cuando hay un núcleo con dos vocales (diptongo) se elimina una de ellas, como se ve en los ejemplos del cuadro 7 :

Cuadro 7. Hipocorísticos con monoptongación

\begin{tabular}{|l|c|}
\hline \multicolumn{1}{|c|}{ Nombre } & Hipocorístico con monoptongación \\
\hline Donaciano & {$[$ 'tfa.no] } \\
\hline Sergio & {$[$ 'tfe.ko] } \\
\hline Ezequiel & {$[$ 'tfe.ke] } \\
\hline Rosario & {$[$ 'tfa.ro] } \\
\hline Graciela & ['tfe.la] \\
\hline Asunción & ['tfo.na $]$ \\
\hline
\end{tabular}

Si en lugar de un diptongo, existe un hiato, entonces no hay simplificación: [tfa.li.o] Rosalío, ['t‘i.o] Rocío.

\section{Mecanismos segmentales}

Los mecanismos de tipo segmental que localicé fueron varios. Los que más se presentaron fueron la palatalización, oclusivización y la asimilación. También surgieron algunos cambios vocálicos, aunque con menor frecuencia.

\section{Oclusivización}

Los casos más frecuentes fueron de $/ \mathrm{f} />/ \mathrm{p} / \mathrm{y} / \mathrm{x} />/ \mathrm{k} /$ en el ataque silábico, tal como se ve enseguida (cuadro 8). 
Hubo algunos otros casos como /'ti.na/ Delfina, Catalina, /'ten.t5a/Inocencia y /'ta.t5o/ Bonifacio, en los que también puede observarse la oclusivización y cambio de punto de articulación: /f/, /l/>/t/.

Cuadro 8. Hipocorísticos con oclusivización

\begin{tabular}{|l|c|}
\hline Nombre & Hipocorístico con oclusivización \\
\hline Alfonso & $/ \mathrm{f} />/ \mathrm{p} /$ \\
\hline Francisco & {$[$ 'pon.tfo] } \\
\hline Josefina & {$[$ 'pan.tfo] } \\
\hline & {$[\mathrm{t}$ e.pi.na] } \\
\hline Jorge & $/ \mathrm{x} />/ \mathrm{k} /$ \\
\hline Alejandra & {$[$ 'ko.ke] } \\
\hline Virginia & {$[$ 'kan.da] } \\
\hline
\end{tabular}

\section{Palatalización ${ }^{7}$}

Este mecanismo puede verse como un caso de oclusivización (más concretamente como de un fenómeno en que una fricativa se vuelve africada), ya que no ocurre únicamente en contextos que favorecen la palatalización (por ejemplo, antes de las vocales $e, i$ ). La consonante que más se palatalizó fue /s/, seguida de /n/. Boyd-Bowman asegura que "La palatalización parece ser un recurso típico del lenguaje afectivo" (1955: 350), y más adelante refuerza esta idea: "En diversas partes del mundo hispánico se ha verificado la palatalización de una sibilante en voces afectivas" (1955: 351).

\footnotetext{
${ }^{7}$ En México, sobre todo en el norte del país, puede darse una "articulación totalmente fricativa de /ch/ con la eliminación del elemento oclusivo: [šs]" (Moreno de Alba, 1994: 119) lo que produce hipocorísticos del tipo ['ta. Jo] Anastasio, ['Sa.ba] Salvador, ['Ja.jo] Santiago, ['Ja.jo] Rosario, ['Je.la] Graciela.
} 
Por lo tanto, los hipocorísticos, como vocablos afectivos, presentan muy comúnmente este fenómeno ${ }^{8}$ (cuadro 9).

Cuadro 9. Hipocorísticos con palatalización

\begin{tabular}{|l|c|}
\hline \multicolumn{1}{|c|}{ Nombre } & Hipocorístico con palatalización \\
\hline Rosario & ['ffa.ro] \\
\hline Rosendo & ['ffen.do] \\
\hline Esperanza & [pe.lan.tfa] \\
\hline Antonieta & [to.'ne.ta] \\
\hline Antonio & ['to.no] \\
\hline
\end{tabular}

\section{Lateralización}

En algunas ocasiones el fonema oclusivo /d/ y el vibrante simple / $/$ / toman el modo de articulación de la lateral /l/: ['la.lo] Eduardo, ['tfi.lo] Isidro, ['lan.tfa] Esperanza, ['le.na] Lorena, ['len.t5o] Lorenzo.

\section{Reduplicación}

En mi trabajo también se produjeron diversos casos de reduplicación (cuadro 10):

Cuadro 10. Hipocorísticos con asimilación

\begin{tabular}{|l|c|c|}
\hline \multirow{2}{*}{ Nombre } & \multicolumn{2}{|c|}{ Hipocorístico con proceso de asimilación a distancia } \\
\cline { 2 - 3 } & De tipo regresivo & De tipo progresivo \\
\hline Sergio & ['ke.ko] & ['tfe.tfo] \\
\hline Bernardo & & ['nan.do] \\
\hline Armando & ['nan.do] & ['ma.mo] \\
\hline Enrique & ['ki.ke] & \\
\hline Federico & ['ki.ko] & \\
\hline Felipe & ['pi.pe] & \\
\hline Socorro & ['ko.ko] & \\
\hline Magdalena & ['ne.na] & \\
\hline Margarita & ['ti.ta] & \\
\hline
\end{tabular}

${ }^{8}$ El caso de los hipocorísticos ['to.no] y [to.'ne.ta], que surgen de los nombres Antonio y Antonieta, podrían verse como casos de fusión debido a la vocal /i/ que se encuentra en adyacencia de la nasal. 


\section{Otros mecanismos}

En algunos casos (muy pocos) se dio una reduplicación de alguna de las sílabas: ['to.to] Evaristo, /lu.'lu/ Lourdes, [t fu'tfu] Jesús. De nuevo, este escaso número no me permite establecer un patrón de comportamiento. Algo similar se dio en el caso de algunos cambios vocálicos. Boyd-Bowman (1955) dijo que por lo general las vocales se mantienen firmes en la creación de hipocorísticos. Y básicamente en mi trabajo así sucedió, salvo contadas excepciones (véase el cuadro 11). Lo interesante es que la columna de la derecha muestra cuatro casos que se dieron en Ciudad Juárez, que colinda con los Estados Unidos. Así que cabe la posibilidad de que dicho cambio haya operado por la cercanía de esta ciudad con nuestro vecino del norte.

\section{Mecanismos morfológicos}

El último bloque de mecanismos se refiere a aquellos que añaden algún tipo de sufijo a los hipocorísticos. En mi investigación localicé principalmente tres: adición de $-i$, adición de $-s$ y adición de $-o,-a$ para indicar el género. Sólo en contadas ocasiones se dio una diminutivización, es decir, un mecanismo en el cual el hipocorístico se forma partiendo del diminutivo del antropónimo.

Cuadro 11. Hipocorísticos con cambios vocálicos

\begin{tabular}{|c|c|c|}
\hline$/ \mathrm{i} />/ \mathrm{e} /$ & /o/ sin marca de género & $\begin{array}{c}/ \mathrm{a} />/ \mathrm{e} / \\
\text { (probablemente por influencia } \\
\text { del inglés) }\end{array}$ \\
\hline $\begin{array}{l}\text { ['be.ri] Viridiana } \\
\text { ['te.mo] Timoteo }\end{array}$ & $\begin{array}{l}\text { ['bi.ko] Virginia } \\
\text { ['ma. }{ }^{\prime} \text { o] Margarita }\end{array}$ & $\begin{array}{l}\text { ['me.me] Manuel } \\
\text { ['te.ße] Esteban } \\
\text { ['me.ri] María } \\
\text { ['se.mi] Samuel }\end{array}$ \\
\hline
\end{tabular}


Adición de -i

En México es común el uso de este sufijo tanto en nombres femeninos como masculinos (cuadro 12).

Cuadro 12. Hipocorísticos con adición de -i

\begin{tabular}{|c|c|c|}
\hline Masculinos & Femeninos & Para ambos géneros \\
\hline $\begin{array}{l}\text { ['fre. ði] Alfredo } \\
\text { ['tu.ri] Arturo } \\
\text { ['ber.ni] Bernardo }\end{array}$ & $\begin{array}{l}\text { ['ro.si] Rosa } \\
\text { ['su.si] Susana } \\
\text { ['na.ti] Natalia }\end{array}$ & $\begin{array}{l}\text { ['sami] Samuel, Samanta } \\
\text { ['mon.tfi] Ramón, Ramona } \\
\text { ['an.di] Andrés, Andrea }\end{array}$ \\
\hline
\end{tabular}

Adición de -s

Boyd-Bowman se refiere a este fenómeno como "al cariñoso trueque de número que representa tal vez la -s" (1955: 360). Por mi parte, no creo que dicha -s tenga relación alguna con el número. Me parece que se trata más bien de un caso de sincretismo, en el que dos morfemas con distinta función poseen una forma idéntica. Pueden verse algunos ejemplos en el siguiente cuadro:

Cuadro 13. Hipocorísticos con adición de -s

\begin{tabular}{|l|l|}
\hline \multicolumn{1}{|c|}{ Masculinos } & \multicolumn{1}{c|}{ Femeninos } \\
\hline ['e.fras] Efraín & ['bi.ßis] Viviana \\
['fa.ßis] Fabián & ['re.xis] Regina \\
['ben.xas] Benjamín & ['kon.Sis] Concepción \\
\hline
\end{tabular}

\section{Adición de género}

Los morfemas -o para el masculino y - $a$ para el femenino fueron utilizados en algunas ocasiones en la creación de hipocorísticos. Se dieron casos como ['tfa.ßo] Salvador,

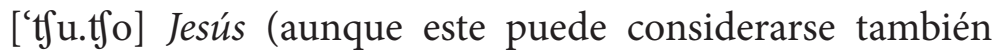




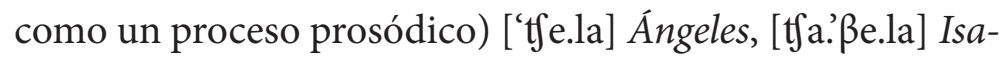
bel, [‘'to.na] Asunción.

\section{Diminutivización}

Los diminutivos no se consideran verdaderos hipocorísticos. No obstante, el morfema de diminutivo aplicado al hipocorístico sí lo tomé como parte de mi trabajo. Se dieron casos como [ta.ßi.to] Gustavo, [fi.ni.ta] Delfina, [kar.me.'li. ta] Carmen, [lo.'li.ta] Dolores, [t 9 a.'ri.to] Rosario y [to.'ni.ta] Antonieta.

A continuación se muestran agrupados (cuadro 14) cada uno de los mecanismos que encontré durante mi trabajo de investigación.

Cuadro 14. Mecanismos utilizados en México

\begin{tabular}{|c|c|c|c|}
\hline Prosódico & Silábico & Segmental & Morfológico \\
\hline $\begin{array}{l}\text { Truncamiento } \\
\text { Reasignación de acento } \\
\text { Elisión de vocal final } \\
\text { Adición de sílaba -ch(o) }\end{array}$ & $\begin{array}{l}\text { Elisión de coda } \\
\text { compleja } \\
\text { Simplificación de } \\
\text { inicio complejo } \\
\text { Monoptongación }\end{array}$ & $\begin{array}{l}\text { Oclusivización } \\
\text { Palatalización } \\
\text { Lateralización } \\
\text { Asimilación } \\
\text { Reduplicación } \\
\text { Cambios vocálicos: } \\
\text { - a > e (por posible } \\
\text { influencia del in- } \\
\text { glés) } \\
\text { - i e e } \\
\text { - o sin marca de } \\
\text { género }\end{array}$ & $\begin{array}{l}\text { Adición de -i } \\
\text { Adición de -s } \\
\text { Adición de gé- } \\
\text { nero } \\
\begin{array}{l}\text { Diminutivi- } \\
\text { zación }\end{array}\end{array}$ \\
\hline
\end{tabular}




\section{Conclusiones}

La finalidad de mi investigación era mostrar no tanto los hipocorísticos sino los mecanismos que se presentan en su creación. Al mismo tiempo se pretendía abarcar una gran parte del territorio nacional, con la finalidad de encontrar el mayor número de mecanismos vigentes en la formación de hipocorísticos. Una vez terminado el trabajo, se pudo llegar a las siguientes conclusiones:

- La mayoría de los mecanismos se presentaron en todas las localidades visitadas. Salvo contadas excepciones como el cambio vocálico de a > e que sólo fue recogido en Ciudad Juárez, la elisión de vocal final que fue un fenómeno que prácticamente sólo se presentó en Mérida y el relajamiento de la africada $/ \mathrm{t} /$ en posalveolar / $/$ / que si bien no es un mecanismo como tal, sí es un fenómeno restrictivo de la zona norte de México.

- Aunque algunos mecanismos fueron de escasa aparición en esta investigación, me parece pertinente incluirlos en alguno de los cuatro grandes bloques de procesos por una simple razón: si aparecieron es porque existen y puede ser que en algún otro momento adquieran mayor vitalidad. Sin embargo, también podría ser que estén cayendo en desuso y por esa razón no fueron tan utilizados por los hablantes.

- Quizá el hecho de que haya tantos fenómenos de tipo segmental se debe al origen infantil de los hipocorísticos ya mencionado por Boyd-Bowman (1955). El habla de un niño modifica inconscientemente los segmentos de las sílabas de diversas maneras: oclusivizando fricativas, pala- 
talizando algunas consonantes, asimilando ciertos fonemas a sus sonidos vecinos y reduplicando determinadas sílabas. Incluso también pueden encontrarse en el habla infantil los mecanismos de corte silábico. Los otros dos tipos de procesos - prosódicos y morfológicos- dependen de factores distintos al habla infantil. También es probable que la gran cantidad de mecanismos se deba a otras cuestiones. Es decir, que quizá el truncamiento a partir de la sílaba inicial no propicia la aparición de tantos procesos mientras que el truncamiento a partir de la sílaba acentuada puede ser un agente disparador de otros mecanismos creadores de hipocorísticos.

\section{Bibliografía}

Báez Pinal, Gloria E. (2002), "Vitalidad y tradición de hipocorísticos empleados en la ciudad de México: 19551999. Un estudio comparativo", Anuario de Letras, XL, pp. 241-265.

—-, María Eugenia Herrera Lima y José Francisco Mendoza (1993), “Antropónimos en el español de la ciudad de México. Tradición y novedad", Anuario de Letras, XXXI, pp. 431-496.

Boyd-Bowman, Peter (1955), "Cómo obra la fonética infantil en la formación de los hipocorísticos", Nueva Revista de Filología Hispánica, 9: 4, pp. 337-366.

Casado Velarde, Manuel (1999), "Otros procesos morfológicos: acortamientos, formación de siglas y acrónimos", en I. Bosque y V. Demonte (eds.), Gramáti- 
ca descriptiva de la lengua española, Madrid, EspasaCalpe, pp. 5075-5096.

Clements, G. N. (1990), "The role of the sonority cycle on core syllabification", en Papers in Laboratory Phonology, Cambridge, Cambridge University Press.

Colina, Sonia (1996), "Spanish truncation processes: The emergence of the unmarked", Linguistics, 34, pp. 11991218.

Espinosa, M. (2001), "De Alfonso a Poncho y de Esperanza a Lancha: Los hipocorísticos”, Razón y Palabra, 21. En línea en http://www.razonypalabra.org.mx/anteriores/ n21/21_mespinosa.html

González Moreno, Jesús (1935), "El español en México", Investigaciones Lingüisticas, III, pp. 171-181.

Gutiérrez Santana, Lucila (2009), Procesos fonológicos utilizados en la formación de hipocorísticos: una aproximación desde la fonología no lineal, Concepción, Universidad de Concepción, Chile.

Henríquez Ureña, Pedro (1921), "Observaciones sobre el español de América”, Revista de Filología Española, VIII, pp. 357-390.

Lope Blanch, Juan M. (coord.) (1990), Atlas lingüístico de México, tomo I, vol. 1, Fonética, México, Universidad Nacional Autónoma de México y El Colegio de México. _ (1981), "Sobre la influencia fonética maya en el español de Yucatán”, Thesaurus. Boletín del Instituto Caro y Cuervo, XXXVI, 3, pp. 413-428.

Moreno de Alba, José G. (1994), La pronunciación del español en México, México, El Colegio de México. 
Moreno de Alba, José G. (1976), "Zonas dialectales de Tabasco y Veracruz. Estudio léxico", Nueva Revista de Filología Hispánica, 25: 2, pp. 332-352.

Núñez Cerdeño, Rafael A. y Alfonso Morales Front (1999), Fonología generativa contemporánea de la lengua española, Georgetown, Washington, D. C., Georgetown University Press.

Ohannesian, Maria (2004), La asignación del acento en castellano, tesis doctoral, Barcelona, Universidad Autónoma de Barcelona.

Prieto, Pilar (1992), "Truncation processes in Spanish", Studies in the Linguistic Sciences, 22, pp. 143-158.

Real Academia Española (2001), Diccionario de la lengua española, 22a ed., Madrid, Espasa-Calpe.

Sempere, Antonio Grau (2006), "(In)sensibilidad a la cantidad silábica en la prosodia portuguesa”, Revista Virtual de Estudos da Linguagem - ReVEL, 4, n. 7.

Serrano Morales, Julio César (2002), “¿Cuántos dialectos del español existen en México? Un ensayo de dialectología perceptual”. En línea en: http://lef.colmex. $\mathrm{mx} /$ Sociolinguistica/Cambio\%20y\%20variacion/Ensayo\%20de\%20dialectologia\%20perceptual.pdf

Urawa, Mikío (1985), "Muestra de hipocorísticos en el español bogotano", Thesaurus, 40, pp. 51-102.

Wijk, Henrik L. A. (1964), "Los hipocorísticos hondureños”, Romanistisches Jahrbuch, 15, pp. 302-312. 
